\section{Why All the Shots at Airway Pressure Release Ventilation When Conventional Ventilation Doesn't Have Consensus?}

\section{To the Editor:}

I would like to thank the authors for bringing to light the need for further education on airway pressure release ventilation (APRV) and how to manage this mode of ventilation. ${ }^{1}$ However, for Miller, Gentile, Davies, and MacIntyre to state that "there is only limited consensus among practitioners for initial APRV settings" and to infer that this is a problem for APRV may be misleading. Generally speaking, in terms of PEEP and ideal volume strategies, there is no overarching common consensus among practitioners on how to accurately set up conventional ventilation either. Had a similar survey been performed asking practitioners where they should set the tidal volume $\left(\mathrm{V}_{\mathrm{T}}\right)$ or PEEP on a conventional ventilator, I believe a similar limited consensus result would have been reached. The same result would occur when asking what mode of conventional ventilation should be used (eg, volume control, pressure control, or pressure-regulated volume control). In fact, it has been reported in a secondary analysis of a multi-center cohort ${ }^{2}$ that there was substantial variability in ventilator settings not entirely explained by subjects' risk for ARDS.

Additionally, this fact is demonstrated by the topics of the June 2016 issue of RESPIRATORY CARE. Within that publication, when asked about randomized, controlled trials, an author stated, "The results really only apply to the specific population studied (often a small fraction of the disease of interest) and only to the specific intervention strategy. This last point is particularly important in complex interventions like mechanical ventilation where potential settings and management strategies can be quite variable." 3

Miller et $\mathrm{al}^{1}$ state that "APRV is not an on-off switch ... as a consequence, interpreting trials and making recommendations about APRV problematic." However, one of the authors in 2016 stated the following about high-frequency oscillatory ventilation (HFOV), "[HFOV] is certainly not an onoff switch and involves not only numerous specific ventilator adjustments but also adjustments in other aspects of management (eg, fluids, pressors) that clearly can impact results. To draw the conclusion that HFOV managed differently in different patients is necessarily harmful seems excessive." ${ }^{3}$ The question now becomes, why do the authors differ in their interpretation of APRV and HFOV not serving as an on-off switch? The evidence reported to date on APRV shows that it is no worse than conventional ventilation in the limited studies that compared the 2 methods, in contrast with a large clinical trial that demonstrated poor outcomes with HFOV. ${ }^{3,4}$

When you look at the authors' ${ }^{1}$ reported survey responses on the $\mathrm{P}_{\text {high }}$ setting, a patient with conventional settings and measurements of PEEP $15 \mathrm{~cm} \mathrm{H}_{2} \mathrm{O}, \mathrm{P}_{\mathrm{pl}}$ $28 \mathrm{~cm} \mathrm{H}_{2} \mathrm{O}$, and peak inspiratory pressure $30 \mathrm{~cm} \mathrm{H}_{2} \mathrm{O}$, the patient would have a mean of approximately $20 \mathrm{~cm} \mathrm{H}_{2} \mathrm{O}$. This would mean that $79 \%$ of the survey respondents would set their $\mathrm{P}_{\text {high }}$ at $25-28 \mathrm{~cm} \mathrm{H}_{2} \mathrm{O}$. Are we really saying that this difference of $3 \mathrm{~cm} \mathrm{H}_{2} \mathrm{O}$ for an initial $\mathrm{P}_{\text {high }}$ setting is that bad and represents a poor understanding of APRV? We accept a range for an initial $\mathrm{V}_{\mathrm{T}}$ in conventional ventilation, so why do we not accept a range for $\mathrm{P}_{\text {high }}$ ? The fact that respondents didn't fit exactly as 2 protocols spelled out does not mean that there is a problem or a lack of understanding.

With regard to the limited consensus findings on $\mathrm{T}_{\text {low }}$, given how the survey question was asked, an initial setting of $0.2-0.8 \mathrm{~s}$ could be a correct statement depending on the reader's interpretation of the question. If a respondent read the question as asking where you would initially set the $T_{\text {low }}$, you have to initially set it somewhere before you find out if it is at $50-75 \%$ of peak expiratory flow (PEF). Even one of the referenced APRV protocols ${ }^{5}$ states "we usually start with a $\mathrm{T}_{\text {low }}$ of $0.6-$ $0.8 \mathrm{~s}$." So, $0.2-0.8 \mathrm{~s}$ is a good place for an initial setting until it is on the patient, and then you adjust it. Therefore, $76 \%$ of the respondents accurately answered the question. Would we get the same type of consensus if you were to ask where to set the initial PEEP in conventional ventilation? The authors cited Amato et $\mathrm{al}^{6}$ as demonstrating that $\mathrm{V}_{\mathrm{T}} \mathrm{S}$ are increasingly appreciated as an independent risk factor for ventilator-induced lung injury. However, Amato et $\mathrm{al}^{6}$ reported that individual changes in $\mathrm{V}_{\mathrm{T}}$ were not independently associated with survival and were only associated with improved survival if the $\mathrm{V}_{\mathrm{T}}$ changes led to decreases in $\Delta \mathrm{P}$. Amato et $\mathrm{al}^{6}$ only mention $\mathrm{V}_{\mathrm{T}}$ separately as an independent risk factor in their introduction and then reference other papers' positions, not their own findings. Additionally, APRV has been found to be able to deliver similar $\mathrm{V}_{\mathrm{T}} \mathrm{S}$ as conventional ventilation with low $\Delta$ Ps. $^{7}$ Via a mathematical lung model, when using APRV on a simulated patient with ARDS, the $\mathrm{V}_{\mathrm{T}}$ achieved was $6.6 \mathrm{~mL} / \mathrm{kg}$ with a total PEEP of $11 \mathrm{~cm} \mathrm{H}_{2} \mathrm{O}^{6}$ The lung model and vent settings resulted in a $\Delta \mathrm{P}$ of $14 \mathrm{~cm} \mathrm{H}_{2} \mathrm{O}$ and achieved the same PEEP as what would be recommend in the ARDSnet PEEP table (10-14 $\mathrm{cm} \mathrm{H}_{2} \mathrm{O}$ ) for the entered $\mathrm{F}_{\mathrm{IO}_{2}}{ }^{7}$ Furthermore, the $\mathrm{T}_{\text {low }}$ in the study was only set for approximately $50 \%$ of PEF. Had the $\mathrm{T}_{\text {low }}$ been shortened to $75 \%$, as proposed by others, ${ }^{8,9}$ the intrinsic PEEP would have been higher, $\mathrm{V}_{\mathrm{T}}$ would have been lower (thus closer to the ARDSnet recommended $6 \mathrm{~mL} / \mathrm{kg}$ ), and the $\Delta \mathrm{P}$ would have been even lower, resulting in a potential decrease in mortality based on Amato et $\mathrm{al}^{6}$ These results demonstrate via a mathematical model that APRV could be seen as in keeping with Amato et $\mathrm{al}^{6}$ and even as current evidence for conventional ventilation.

Regarding PEEP and APRV, PEEP is another area of limited consensus. However, the different levels, high versus low, have not been shown to demonstrate changes in the outcomes of patients with ARDS. ${ }^{10}$ Therefore, APRV is being compared to something that also has no consensus. A recent study has demonstrated that using a $\mathrm{T}_{\text {low }}$ of $75 \%$ of PEF has been found to produce less micro-strain and macro-strain on alveoli versus $\mathrm{PEEP}$ values of $5 \mathrm{~cm} \mathrm{H}_{2} \mathrm{O}$ or $16 \mathrm{~cm} \mathrm{H}_{2} \mathrm{O} .{ }^{8}$ Furthermore, setting PEEP to higher levels has been reported to create significantly different alveolar size distribution within the lung versus setting a $\mathrm{T}_{\text {low }}$ to $75 \%$ of PEF. ${ }^{9}$ So while a set PEEP may or may not generate a consistent end-expiratory pressure in the alveoli, it has been shown to create more strain and greater variance in alveolar size. Which is worse for the lung? Given the lack of data demonstrating a survival benefit for higher versus lower PEEP in conventional ventilation, maybe it's time we start to look at another way to stabilize the lung. A set PEEP value doesn't seem to be the answer, and if it is, the evidence has not shown us how to set it correctly.

The article by Miller et $\mathrm{al}^{1}$ is based on 60 responses and was limited to 1 per institution from individuals in the AARC Adult Acute Care Section, which has approximately 1,900 members. This represents $3 \%$ of that membership and an even lower percentage of practicing therapists. In addition, given the fact that the authors limited the number of responses to 1 per institution, it really only assesses the first person to see and respond to the survey versus a true as- 
sessment of how clinicians set up and manage APRV. With a $3 \%$ representation of a small subpopulation of therapists, can we truly assess how bedside clinicians are using APRV from this survey?

Why should APRV be held to a different standard than conventional ventilation or HFOV? We can't reach consensus on any ventilator settings, so why should/would APRV be any different? I also suggest that the agreement on APRV settings among clinicians might actually be higher than what would be found in conventional ventilation or HFOV, given the high degree of scrutiny it has endured over the years.

\section{Aaron Light DHSc RRT-ACCS} Allied Health

Ozarks Technical Community College Springfield, Missouri

Dr Light discloses a relationship with Dräger.

DOI: $10.4187 /$ respcare. 05980

\section{REFERENCES}

1. Miller AG, Gentile MA, Davies JD, MacIntyre NR. Clinical management strategies for airway pressure release ventilation: a survey of clinical practice. Respir Care 2017;62(10):1264-1268.

2. Chang SY, Dabbagh O, Gajic O, Patrawalla A, Elie MC, Talmor DS, et al.; United States Critical Illness and Injury Trials Group: Lung Injury Prevention Study Investigators (USCIITG-LIPS). Contemporary ventilator management in patients with and at risk of ALI/ARDS. Respir Care 2013; 54(4):578-588.

3. Nguyen AP, Schmidt UH, MacIntyre NR. Should high-frequency ventilation in the adult be abandoned? Respir Care 2016;61(6):791800.

4. Jain SV, Kollisch-Singule M, Sadowitz B, Dombert L, Satalin J, Andrews P, et al. The 30-year evolution of airway pressure release ventilation. Intensive Care Med Exp 2016;4(1):11.

5. Modrykamien A, Chatburn RL, Ashton RW. Airway pressure release ventilation: an alternative mode of mechanical ventilation in acute respiratory distress syndrome. Cleve Clin J Med 2011;78(2):101110.

6. Amato MB, Meade MO, Slutsky AS, Brochard L, Costa EL, Schoenfeld DA, et al. Driving pressure and survival in the acute respiratory distress syndrome. $\mathrm{N}$ Engl J Med 2015;372(8):747-755.

7. Sasidhar M, Chatburn RL. Tidal volume variability during airway pressure release ventilation: case summary and theoreti- cal analysis. Respir Care 2012;57(8): 1325-1333.

8. Kollisch-Singule M, Emr B, Smith B, Roy S, Jain S, Satalin J, et al. Mechanical breath profile of airway pressure release ventilation. JAMA Surg 2014;149(11): 1138-1145.

9. Kollish-Singule M, Jain S, Andrews P, Smith BJ, Hamlington-Smith KL, Roy S, et al. Effect of airway pressure release ventilation on dynamic alveolar heterogeneity. JAMA Surg 2016;151(1):64-72.

10. Hess DR. Recruitment maneuvers and PEEP titration. Respir Care 2015;60(11): 1688-1704.

\section{Airway Pressure Release Ventilation Letter-Reply}

We thank Dr Light for his insights on airway pressure release ventilation $(\mathrm{APRV})^{1}$ and will address his comments one by one below. However, we first re-emphasize that the purpose of our study ${ }^{2}$ was not to address the clinical value of APRV - that can only be accomplished with randomized clinical trials. In contrast, our goal was to illustrate the current practice of experienced clinicians using APRV and to point out that APRV is not a simple "on-off" switch; rather, it utilizes 4 non-conventional settings that can be adjusted over wide ranges. We believe we have shown that there is considerable practice variability among experienced APRV users when using the mode, and that there is the potential for untoward consequences.

Our thoughts on the specific points raised by Dr Light:

With regard to conventional ventilation settings, while we agree that settings for conventional mechanical ventilation (loosely defined as mimicking the normal breathing pattern) often lack consensus, there is a considerable evidence base driving consensus on ventilator management of the acutely injured lung. Starting with the ARDS Network's small tidal volume $\left(\mathrm{V}_{\mathrm{T}}\right)$ trial $^{3}$ and followed by several subsequent trials, ${ }^{4}$ a strong consensus has emerged to limit $\mathrm{V}_{\mathrm{T}} \mathrm{S}$ and the end-inspiratory airway plateau pressures to physiologic ranges. Recent large sur- veys indicate that most ICUs have adopted this approach. ${ }^{5}$

With regard to similarities between APRV and high-frequency oscillatory ventilation (HFOV), we agree that similarities exist between APRV and HFOV and thank Dr Light for highlighting this point. Both strategies are based on CPAP principles, and both manipulate CPAP in non-conventional ways: APRV with periodic brief releases, HFOV with superimposed small-amplitude oscillations. Moreover, with both modes there are nonconventional settings that can be manipulated in multiple ways that, depending on patient characteristics, can clearly affect outcomes. Underscoring this point is the recent metaanalysis of adult randomized clinical trials demonstrating that, while HFOV may be an effective rescue strategy in very severe lung injury, it can cause considerable harm when delivered inappropriately to subjects doing well on conventional ventilation. ${ }^{6}$

With regard to the $\mathrm{P}_{\text {high }}$ setting, given that existing APRV guidelines 7,8 recommend limiting $\mathrm{P}_{\text {high }}$ to $<30-35 \mathrm{~cm} \mathrm{H}_{2} \mathrm{O}$ (in agreement with many conventional ventilation guidelines), we were concerned that $36 \%$ of our respondents accepted values above that level. This result also heightened our concern that many respondents may not fully appreciate that spontaneous efforts occurring during $\mathrm{P}_{\text {high }}$ will add to the maximal transpulmonary pressure, further increasing the risk of lung injury.

With regard to the $\mathrm{T}_{\text {low }}$ setting, while it is always possible that respondents may have misunderstood our question regarding the initial $\mathrm{T}_{\text {low }}$ setting, we believe that most would interpret our question to be addressing the setting at which the patient is started and then is followed for a period of time before reassessing. We stand by our interpretation that there is limited consensus on whether to use an absolute time setting or to use a variety of expiratory flow analyses to set $\mathrm{T}_{\text {low }}$. 